\title{
Sub-pixel Distance Measurement Algorithm Based on Improved SURF

\author{
Zixiang Fu
}

College of Science, Tianjin Polytechnic University, Tianjin, 300387, China

\begin{abstract}
A sub-pixel-level measurement algorithm based on improved SURF is proposed. Firstly, the sobel operator is used to extract the edge of the image quickly in order to highlight the potential feature point, and reduce the detected range of the SURF algorithm. Then use the SURF algorithm to obtain the sub-pixel level coordinates of the matching feature points in the two images. After the match, the affine transformation remove the wrong match point. Finally, the moving distance is calculated by using the sub-pixel-level coordinates of the matching points. The experimental results show that the error of the moving distance is less than $3 \%$, according to the feature points of the two images. And if the original image resolution is $2560 * 1440$, the required running time is only 0.3 to 0.4 seconds.
\end{abstract}

Keywords-Distance Measurement, SURF, Sub-pixel, Sobel.

\section{INTRODUCTION}

At present, there are many image-based measurement distance algorithms have been proposed. These image-based ranging methods can be roughly divided into two categories. In the first categories, the image obtained by a single camera is processed to measure distance. For example, Suraphol proposed to measure the length of an object [3] by using the acceleration signals and object differences. Han et al. used the highest feature point of the marker crown and the triangular similarity theory to measure the length [4]. Zhao et al. proposed to obtain the centroid of the odd and even field by using the interval scan to measure speed [5]. The second category is through two cameras to obtain images, using the difference between the two images to calculate the distance. For example, Yasir et al. used visual differences and camera focal lengths from two parallel cameras to measure distance [6]. Hou A-Lin built stereoscopic vision with two cameras and then calibrates with the projection matrix to measure the safe distance traveled by the vehicle [7]. Hai-Sung Baek proposed to use two cameras to build stereoscopic vision. Through the difference between the two images calculated in a larger field of view, a more accurate measurement of object distance is got [8].

The first categories are not only the image processing, in most cases also need to utilize laser, ultrasonic and other auxiliary equipment to obtain relevant information. The second categories require multiple devices to coordinately use, with more complex hardware devices. Thus, a new distance measurement based on single image is proposed in this paper, which is a more economical and convenient measurement manner.

The SURF algorithm is generally used for matching, comparing and tracking of target objects in various scenarios. Its advantage is that it can quickly locate the coordinates of the feature points, and the coordinates can be subpixel [11-14]. In this paper, the Sobel edge extraction is utilized to reduce the scope of feature extractions, and affine transformation is utilized to avoid false matching result of the SURF algorithm. Afterwards, the SURF algorithm will be used to extract the feature points before and after the target object is moved. Then the result of feature extractions is calibrated by affine transformation. Combined with the angle information, calculate the average of the components from multiple groups measured length values in the direction of motion, and utilize this average value to estimate the distance. The innovation of this paper is to apply the SURF matching algorithm to distance measurement. It not only can quickly and stably obtain the result of the measurement, but also the coordinates of the measured feature point which can reach the sub-pixel precision.

\section{MeTHODOLOGY}

Step1: First at all, the two images of the natural scene along a certain direction of movement are shot. It is required that is overlap or have the same part, because the next algorithm will be based on the characteristics of the two images to the estimated moved distance. When shooting images, it could not take into account the effects of light, allowing a small angle of rotation, and allowing a slight change in height, but as far as possible to keep the image shot in the same depth of field. The original image resolution of is $2560 * 1440$. Taking into account the actual shooting of the image may be too large, so the 
appropriate reduction is needed to improve efficiency. The following results are obtained at a compression ratio of 0.2 (the problem of compression ratio will be discussed in detail below). The default direction of movement is horizontal, and the measured distance is also in this direction. In the actual measurement, the height of the camera determines the camera to capture the scope of view. Fig. 1 shows the results of the algorithm in camera scope of view. And the algorithm can still be stable to obtain the test results, even in different backgrounds and different light.
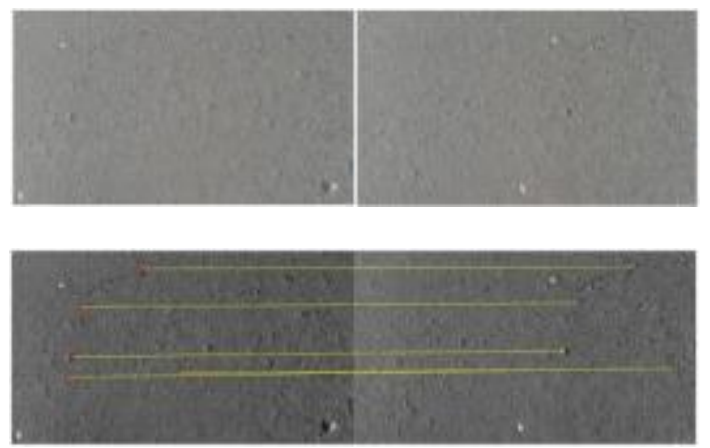

\section{FIG. 1 THE TOP ROW IS THE ORIGINAL IMAGE, THE BOTTOM ROW IS THE MATCHING RESULTS, THE BACKGROUNDS IN ORIGINAL IMAGES ARE: CEMENT ROAD.}

Step 2: In order to reduce the computational complexity of the SURF algorithm, it needs to firstly utilize the sobel operator to extract the edge of the image, which will be also more conducive to extract feature points for the SURF algorithm. This paper chooses Fig1 to extract the edges. The results are shown in Fig. 2.
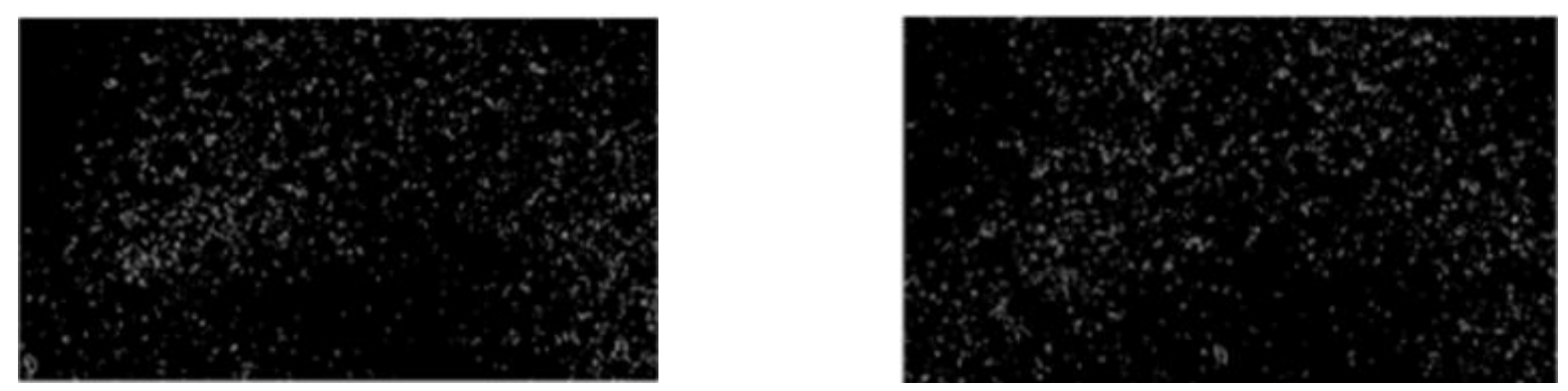

FIG.2. EXTRACT THE EDGES FROM THE ORIGINAL IMAGE

Step 3: After the images have been extracted the edge, the SURF algorithm is used to match the image to find the feature points quickly. After matching, multiple sets of matching feature points will be obtained. Fig. 3 shows the obtained results according to Fig. 2. For Fig. 2, the picture is a combination of two images, the red pixels stand for before moving, green pixels stand for after moving, the red circle stand for feature points of the image before moving (matched point 1 ), the green cross stand for feature points of the image after moving (matched point2). Finally, connect the matching feature points in two images with a yellow line.

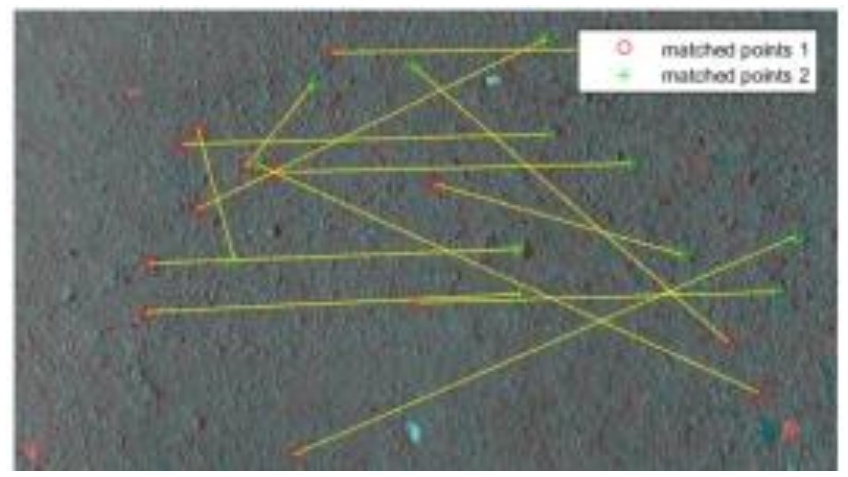

FIG. 3. MATCH THE IMAGES

Step 4: After the images matched, the affine transformation is utilized to calculate the transformation associated with the matching point. This conversion allows us to filter the matched results in two images. The result is shown as Fig. 4. 


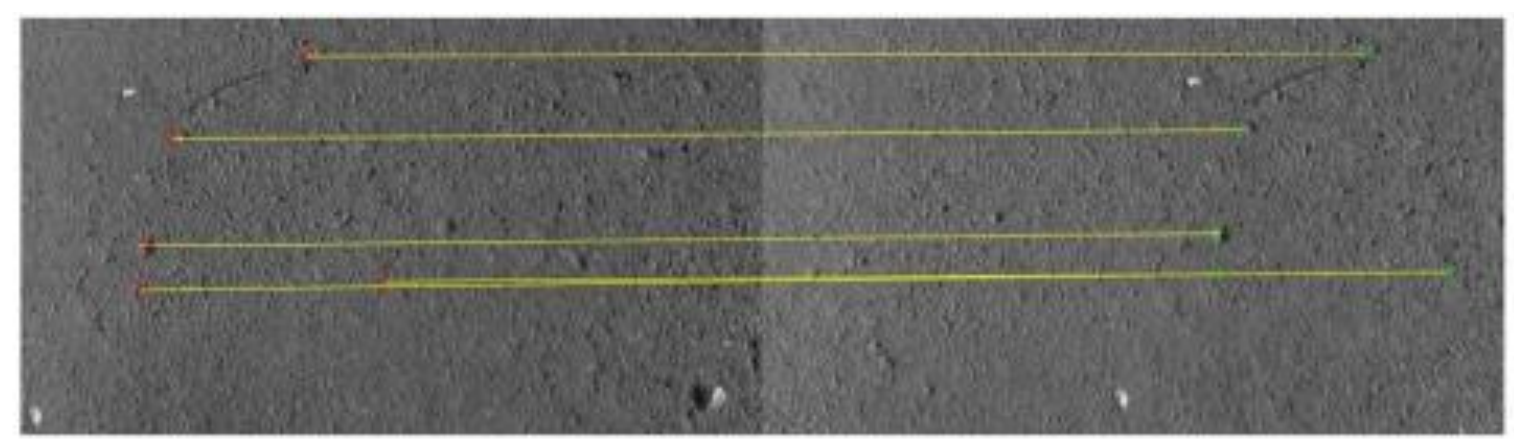

Fig. 4. FilTER MATCHED RESULTS

Step 5: After the SURF algorithm, the coordinates of these feature points have reached the sub-pixel level. Next, the corresponding vectors will be calculated by using these coordinates. Each pair of matching successfully points has two coordinates, so it can get a vector, which contains two information's, namely the length and the angle. It is considered that the direction of the vector formed by matching the feature points cannot be exactly the same as the horizontal direction. Therefore, it is necessary to calculate the angle $\theta$ between the residual vector and the direction of motion (namely, the horizontal direction) to make the results as accurate as possible. In order to eliminate the impact of MSAC algorithm, this paper needs to calculate the average of the length value $\bar{d}$ and the average of the angle value $\bar{\theta}$. Here, the length of vector is $d$. The number of vectors is $n$ and there are:

$$
\begin{gathered}
\bar{d}=\frac{\sum_{i=0}^{n} d_{i}}{n} \\
\bar{\theta}=\frac{\sum_{i=0}^{n} \theta_{i}}{n}
\end{gathered}
$$

Step 6: The length value $\bar{d}$ is multiplied by the cosine of $\bar{\theta}$ to obtain the length component $\bar{w}$ of the vector in the direction of motion. The result is:

$$
\bar{w}=\bar{d} \cos \bar{\theta}
$$

Here, as the image is reduced, it is necessary to divide by the corresponding reduction ratio $k$. This result $w$ is the displacement in the pixel sense $w$ is:

$$
w=\frac{\bar{w}}{k}
$$

Step 7: The image pixel length $h l$ and the actual length $h 2$ are known, the actual moving distance $s$ is obtained from the average value $w$. If the shooting time of two images is known, the speed can be further measured. So the moving distances is:

$$
\frac{w}{s}=\frac{h_{1}}{h_{2}}
$$

\section{EXPERIMENTAL RESULTS}

In order to accurately measure the accuracy of the algorithm, move the camera in parallel at a fixed height. For the distance of $20 \mathrm{~cm}, 9$ sets of data are measured. Fig. 5 shows the line graph of the standard distance and the measured distance for each movement. As it can be seen from Fig. 5, the relative error measured is less than $3 \%$. In the actual movement of the process, due to man-made reasons, the camera's viewing angle and height may be a slight change, which will naturally have an impact on the measurement results. Therefore, the actual error is less than $3 \%$. 


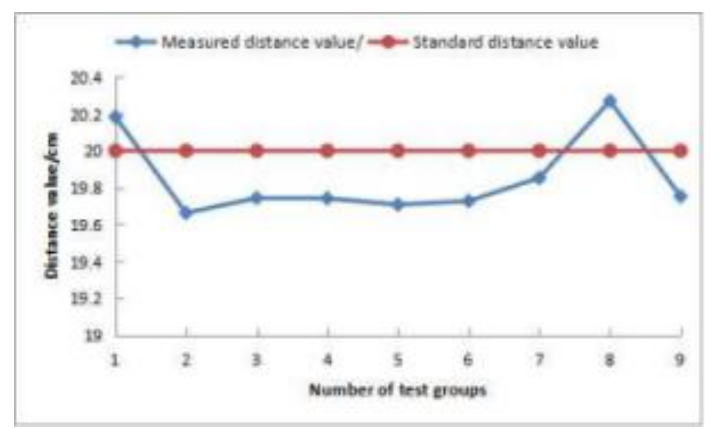

Fig. 5. THE RESULT IS MEASURED WHEN CAMERA MOVING $20 \mathrm{~cm}$ EACH TIME.

IV. CONCLUSION

This paper presents a subpixel distance measurement algorithm. It has rotational invariance, scale invariance, and satisfies the velocity measurement in strong light and low light. The experimental results show that according to the feature points of the image, the error between the measured distance and the actual moving distance is less than $3 \%$ and the time is only 0.3 to 0.4 seconds. The measurement results are retained to 0.0001 . The algorithm is applied to the measurement of distance, and can also be used to measure the speed. The algorithm can be used for the speed measurement of the moving objects in a quiescent state. It can also be made on the vehicle equipment, to measure its speed.

\section{REFERENCES}

[1] L. Suraphol, W. Akarapas, and P. Karn, "Measurement of Size and Distance of Objects Using Mobile Devices", in: Proceedings 2013 International Conference on Signal-Image Technology and Internet-Based Systems, SITIS 2013, 2013, pp.156-161.

[2] D. Y. Han, C. D. Wang, "Tree Height Measurement based on image processing Embeded in Smart Mobile phone", in: 2011 International Conference on Multimedia Technology, 2011, pp. 3293 - 3296.

[3] P. Zhao, "Precise speed measurement using an interlaced scan image", Optics \& Laser Technology,vol.43, 2011, pp. $204-207$.

[4] Y. M. Mustafah, R. Noor, H.h Hasbi, A. W. Azma, "Stereo Vision Images Processing for Real-time Object Distance and Size Measurements", in: 2012 International Conference on Computer and Communication Engineering (ICCCE) , 2012, pp.659 - 663.

[5] A. Hou, X. Cui, Y. Geng, W.J. Yuan, and J. Hou, "Measurement of Safe Driving Distance Based on Stereo Vision”, in: 2011 Sixth International Conference on Image and Graphics, 2011 , pp. 902 - 907.

[6] H.S. Baek, J.M. Choi, and B.S. Lee, "Improvement of Distance Measurement Algorithm on Stereo Vision System (SVS)", in: Proceedings of the 5th International Conference on Ubiquitous Information Technologies and Applications, 2010, pp. 1-3.

[7] Y. Luo, Y.Z. Chen, "Robust matching algorithm based on SURF", in: 2015 12th International Computer Conference on Wavelet Active Media Technology and Information Processing (ICCWAMTIP), 2015,pp.7- 10.

[8] W. Saelao, S.t Wangsiripitak,and C. Pluempitiwiriyawej, "Feature-based motion detection and tracking on approximate 3D ground plane", in: 2016 Visual Communications and Image Processing (VCIP), vol.201,2016, pp. 1 - 46.

[9] L. Xia, Y.G. Gao, and X.D. Han, "Novel theory for propagation of tilted Gaussian beam through aligned optical system", Optics Communications, vol. 387, 2017, pp. 281-289.

[10] P. Chen, F. Chen, Y.X Han, and Z.S Zhang, "Sub-pixel dimensional measurement with Logistic edge model”, Optik , vol. 125,2014,pp. 2076-2080. 